\title{
¿PODER DURO O PODER BLANDO? \\ EL GABINETE DEL PRESIDENTE \\ DEL GOBIERNO EN ESPAÑA
}

\author{
Hard power or soft power? \\ The Office of the President \\ in the Government of Spain
}

\author{
ANTONIO GARRIDO \\ Universidad de Murcia \\ agarrido@um.es \\ M. ${ }^{a}$ ANTONIA MARTÍNEZ \\ Universidad de Murcia' \\ antoniam@um.es
}

Cómo citar/Citation

Garrido, A. y Martínez, M. ${ }^{a}$ A. (2018).

¿Poder duro o poder blando?

El Gabinete del presidente del Gobierno en España.

Revista de Estudios Políticos, 180, 163-195.

doi: https://doi.org/10.18042/cepc/rep.180.06

\section{Resumen}

Este artículo presenta una investigación original que pretende evaluar el funcionamiento dinámico y político, no estrictamente formal, del Gabinete de la Presidencia del Gobierno, conocido popularmente en España como «la Moncloa» o «el complejo de la Moncloa». Para ello, se presenta, por vez primera, una aproximación a su forma de trabajo desde la perspectiva de los distintos jefes del Gabinete (chief of staff) que han dirigido este órgano, mediante un conjunto de entrevistas que abarca a la práctica totalidad de los mismos e incluye también a uno de los presidentes del Gobierno. El objetivo es comprender, desde este doble ángulo, los dos vértices de la

1 Los autores desean agradecer expresamente las observaciones formuladas por los evaluadores de la revista, que han contribuido al mejor desarrollo de distintos aspectos de la investigación original. 
relación entre el primer ministro y su principal instrumento de información y asesoramiento, abordando el surgimiento y la expansión de este órgano en el Gobierno español, su génesis y transformación, a partir de documentos inéditos de los archivos de la Presidencia del Gobierno y, por último, analizando -en la práctica- la estructura, las competencias y las funciones del Gabinete en el proceso de decision-making.

\section{Palabras clave}

España; Presidencia; Gabinete de Presidencia; Gobierno; primer ministro.

\section{Abstract}

This article presents original research that seeks to evaluate the dynamic and political, although not strictly formal, functioning of the Office of the Presidency of the Government, popularly known in Spain as "Moncloa" or "the complex of Moncloa". To this end, it presents for the first time the perspectives of the different heads of the Office (chiefs of staff), investigating the way they work through a set of interviews that also includes one of the presidents of the Government. The objective is to understand, from this double angle, the two vertices of the relationship between the prime minister and his main source of information and advice. The paper addresses the emergence and expansion of this body in the Spanish Government, its genesis and transformation, using unpublished documents from the archives of the Presidency of the Government and, finally, by analyzing -in practice- the structure, powers and functions of the Office in the decision-making process.

\section{Keywords}

Spain; presidency; institutional presidency; presidential office; core executive; cabinet; Moncloa office; president's and prime ministers' chief of staff. 


\section{SUMARIO}

I. INTRODUCCIÓN. II. LA TEORÍA DE LA DELEGACIÓN Y LA FUNCIÓN DE LOS GABINETES PRESIDENCIALES. III. LA CONSTRUCCIÓN DEL GABINETE PRESIDENCIAL Y DEL "COMPLEJO DE LA MONCLOA» DESDE LOS MODELOS COMPARADOS. IV. PODER DURO VS. PODER BLANDO: ESTRUCTURA, COMPETENCIAS Y FUNCIONES DEL GABINETE: 1. Los niveles de la formalidad. 2. El poder blando del Gabinete y la fluidez de la informalidad. 3. La pérdida de agencia y el control ministerial como premisa de la estructura organizativa del Gabinete. V. CONSIDERACIONES FINALES. Bibliografía. Anexos.

\section{INTRODUCCIÓN}

La investigación sobre los gabinetes de los primeros ministros está aumentando en el ámbito del derecho y la política comparada (Rhodes y Tiernan, 2014a y 2014b; Tiernan y Pfiffner, 2014; Truswell y Atkinson, 2011). Este renovado interés gozaba de una notable tradición en algunos casos, como los estudios sobre el staff y el funcionamiento de la Casa Blanca en Estados Unidos; un interés amplificado por la atención de los medios de comunicación, películas y series de televisión (Cohen et al., 2012; Pfiffner, 1993 y 2011; Sullivan, 2004; Virgala Foruria, 1994; Walcott y Hult, 2005). Esta atención no se ha extendido al caso español, que cuenta con escasos estudios sobre su composición, funciones, competencias o estructuras (Molina, 1998; Olías, 1994; Ortega, 1991; Prats i Català y Villoria, 2011). Los trabajos existentes en nuestro país se han referido a los gabinetes de los primeros Ejecutivos de la democracia, por lo que la información disponible no ha sido renovada ni actualizada, o bien han hecho un hincapié muy pronunciado en los aspectos jurídicos, de su regulación, o en los sociológicos, dejando de lado el estudio de su funcionamiento práctico y la influencia política (soft power) que despliegan, además de una discusión en profundidad de su papel en la política española moderna.

La escasa atención académica e investigadora no se corresponde con el rango ni con la importancia política del Gabinete. En otros países, el reconocimiento al papel de primer nivel de los asesores directos del presidente está ampliamente reconocido: en Estados Unidos, James Baker solía señalar que el jefe de Gabinete es la segunda posición más poderosa en el Gobierno, y en un reciente libro, titulado elocuentemente The Gatekeepers: How the White House 
Chieffs of Staff Define Every Presidency, se concluye que «el destino de cualquier presidencia depende de esta posición poco comprendida" (Whipple, 2017: 11); en Alemania, la revista The Economist suele referirse al jefe de la Cancillería o Bundeskanzleramt como «el hombre más poderoso de Berlin», etc.

Dado el papel clave de este órgano, poco estudiado, en este artículo nos centraremos en evaluar el funcionamiento dinámico y político, no estrictamente formal, del Gabinete de la Presidencia del Gobierno, conocido popularmente en Espańa como «la Moncloa» o «el complejo de la Moncloa». Para ello, presentaremos una aproximación a su forma de trabajo desde la perspectiva de los distintos jefes del Gabinete que han dirigido este órgano, mediante un conjunto de entrevistas que abarca a la práctica totalidad de los mismos e incluye también a uno de los presidentes del Gobierno. El objetivo es comprender, desde este doble ángulo, los dos vértices de la relación entre el primer ministro y su principal instrumento de información y asesoramiento: el Gabinete del presidente.

El artículo se divide en tres secciones. En la primera esbozaremos el marco teórico y analítico que explica el surgimiento y la expansión de este tipo de órganos en las democracias contemporáneas. El segundo describe el modelo español del Gabinete del presidente del Gobierno y su génesis a partir de documentos inéditos. El último epígrafe analiza la estructura, las competencias y funciones de este órgano, de acuerdo con una práctica consolidada que es descrita por los propios artífices de las transformaciones y cambios que «el complejo de la Moncloa» ha experimentado en los últimos cuarenta años.

\section{LA TEORÍA DE LA DELEGACIÓN Y LA FUNCIÓN DE LOS GABINETES PRESIDENCIALES}

En los años noventa la aparición del enfoque centrado en el core executive, a partir de los estudios de Rhodes y Dunleavy (1995), desplazó el debate de la discusión del poder del primer ministro frente al poder del Gabinete a otra centrada en el conjunto de instituciones del Ejecutivo que constituyen el centro del Gobierno, a sus mecanismos de coordinación y a la eficacia derivada de los mismos en la implementación de políticas públicas. Las distintas variantes de esta perspectiva analítica, como el asymmetric power model o el resource-dependence approach, e incluso la literatura sobre "presidencialización" del core executive, aplicados a los casos del Reino Unido, Alemania, Francia, Japón o Australia, prestaron una escasa atención al estudio monográfico de ciertos órganos clave del mismo, y especialmente a los gabinetes de los primeros ministros (Burch y Halliday, 2004; Elgie, 2011; Heffernan, 2003; Marsh et al., 2003; Poguntke y Webb, 2005). Algunos de aquellos pioneros estudios sobre policy-making en el poder ejecutivo, no obstante, sí que pusieron de relieve la necesidad de estudiar 
tanto las relaciones de intercambio de recursos entre los diversos actores políticos que forman parte del core executive como la influencia relativa del core executive - y su capacidad de dirección y coordinación política— en el contexto más amplio del sistema de gobernanza, dos aspectos que deben orientar la investigación sobre los gabinetes presidenciales y las llamadas «oficinas» de los primeros ministros (Heywood y Molina, 2000; Müller Rommel, 1994).

Solo la reciente aparición, en el ámbito español y latinoamericano, de una nueva perspectiva de análisis relativa al denominado "centro presidencial» ha generado una mayor atención académica hacia los órganos directos de asesoría de los primeros ministros o los presidentes (Camerlo, 2014; Inácio y Llanos, 2015; Lanzaro, 2016; Méndez, 2014). Dado el carácter muy incipiente de estos estudios, aún no disponemos de un modelo comparado de funcionamiento de los centros presidenciales ni del core executive, y de sus componentes, en nuestras democracias. Por ello, para comprender el surgimiento de algunas de las instituciones, como los gabinetes, que forman parte del core executive o de los centros presidenciales conviene recurrir a algunas teorías analíticas bien consolidadas en los estudios institucionales, como la teoría de la delegación o la teoría de la agencia.

Desde esta perspectiva, las constituciones establecen dos modelos de estructuración de las relaciones entre agentes (los ciudadanos) y principales (sus representantes políticos) en una democracia. En el sistema parlamentario, a través de una cadena singular, única y jerárquica que enlaza a cada agente con un principal superior inmediato; los ciudadanos son los principales o mandantes de los parlamentarios que componen la asamblea y estos, a su vez, son los principales o mandantes del Gobierno que, en estos sistemas, constituye un comité de las Cámaras parlamentarias. En el caso del presidencialismo, mediante dos agentes que tienen que implicarse en constantes intercambios y transacciones horizontales (los ciudadanos son los principales de dos agentes, el presidente y la asamblea).

A su vez, los presidentes y primeros ministros disponen de una serie de agentes para ejecutar sus decisiones e implementar sus políticas. Dentro de esta cadena de delegaciones, los jefes de Gobierno, para reducir las pérdidas de agencia y los costes de control, inducir a los agentes a actuar en beneficio del principal, fiscalizar sus actos y omisiones y evitar que la delegación se transforme en abdicación, pueden utilizar distintas alarmas y procedimientos: ex ante pueden usar técnicas como el cuidadoso diseño de los procedimientos de delegación (o del contrato de delegación) y un riguroso control en el proceso de selección de sus agentes; ex post suelen recurrir a los mecanismos de control e información sobre las actividades de los agentes y a los controles y contrapesos institucionales.

El desarrollo de los modernos gabinetes o centros presidenciales constituye una perfecta aplicación de los procedimientos ex post. Su surgimiento 
implica que hay múltiples agentes del presidente, que deben formular la legislación y las políticas a aplicar, así como vigilar el cumplimiento del programa del partido. Estos distintos agentes (Gobierno, ministros y miembros del Gabinete) se controlan mutuamente y los desacuerdos entre ellos proporcionan al principal, el presidente del Gobierno, la información necesaria sobre su funcionamiento, actuando el Gabinete como la clásica alarma que advierte al principal de las medidas, en detrimento de sus intereses políticos, que han sido adoptadas por sus ministros y otros colaboradores. Esta visión está asociada al proceso de "presidencialización» que han experimentado muchas democracias parlamentarias occidentales, como la española (Heywood y Molina, 1997 y 2000; Paniagua, 2017; Van Biezen y Hopkin, 2005).

El trabajo del Gabinete pone también énfasis en los mecanismos ex ante de selección de agente y en el estudio previo de sus perfiles, con el objeto de evitar que impresiones erróneas acerca de la información correcta sobre los candidatos idóneos para determinados puestos gubernamentales puedan ocasionar pérdidas de agencia. El presidente, con la información que le proporciona su propio Gabinete, selecciona los candidatos adecuados para formar el Gobierno.

\section{LA CONSTRUCCIÓN DEL GABINETE PRESIDENCIAL Y DEL «COMPLEJO DE LA MONCLOA» DESDE LOS MODELOS COMPARADOS}

Dos modelos de la organización del Gabinete pueden considerarse las clásicas fórmulas parlamentarias de referencia, oscilando desde la escasa relevancia o las reducidas dimensiones del Prime Minister Office inglés, con su marcado carácter funcionarial, a la más amplia extensión de personal propia de la Bundeskanzleramt o Cancillería Federal, el Gabinete alemán (aunque también con una notable impronta funcionarial — como el Gabinete sueco-) y, especialmente, del Gabinete francés (con un carácter mixto, mezcla de elementos funcionariales y políticos) que, además, aparece desdoblado en la diarquía presidente-primer ministro, característica de los sistemas de gobierno semipresidencial (Müller-Rommel, 1994). Estos modelos europeos no llegan, en ningún caso, a alcanzar la hipertrofia, tanto en dimensión como en su composición - más política-, del tercer modelo, o tipo ideal de Gabinete, que constituye el caso paradigmático de estas estructuras políticas de asesoramiento del poder ejecutivo: la Casa Blanca (White House) y la Oficina del presidente de los Estados Unidos (Executive Office of the President). Dentro de las democracias parlamentarias tipo Westminster, se aproximan más a este tercer modelo los casos de Australia, Canadá o Nueva Zelanda. 
El «complejo de la Moncloa» empieza a construirse con el nombramiento de Adolfo Suárez como presidente del Gobierno, si bien el Gabinete de los dos primeros jefes del Gobierno de la democracia era muy reducido (véase la Tabla 2 para una relación de los directores del Gabinete y los secretarios generales de presidencia durante la democracia) ${ }^{2}$. La limitación de medios personales y de recursos quizá es la característica más acusada de los gabinetes del presidente Suárez. La Presidencia del Gobierno, en su etapa de la casa-palacio del Paseo de la Castellana, y, especialmente, durante el período estricto de transición entre 1976 y 1977, se limitaba a una jefa del Gabinete, Carmen Díez de Rivera, y a una estructura de personal muy reducida $^{3}$. En esta etapa, el Gabinete "no era un contrapeso de los ministerios» ni actuaba como un segundo agente para limitar ex post las pérdidas de agencia en los ministros que todo proceso de delegación de poder produce, sino que hacía las funciones de «enlace» entre los ministerios y el presidente del Gobierno ${ }^{4}$. Con el traslado del presidente Suárez al Palacio de la Moncloa se introdujeron leves cambios, como el nombramiento de un nuevo jefe de

2 El propio presidente Calvo-Sotelo (1990: 175) recoge, de manera crítica, los diferentes modelos de Gabinete que sustentaban ambas experiencias en las etapas iniciales de la democracia en nuestro país: «En los tiempos de Suárez y en los míos rodeaban al Presidente media docena de personas: hoy cercan a Felipe González más de un centenar [...]. En los tiempos de Suárez y en los míos se hablaba, impropiamente, del Palacio de la Moncloa... ahora se habla ya del complejo de la Moncloa: tanta es la burocracia presidencial [...] En el crecimiento espectacular del complejo de la Moncloa no sólo hay que ver una tendencia muy socialista a aumentar la burocracia, sino algo más: un concepto diferente de la organización presidencial misma [...]. Existen dos modelos de organización en la cumbre de una estructura importante, sea pública o privada: en uno de ellos el Presidente se apoya sobre la llamada línea ejecutiva, es decir, en el caso del Gobierno sobre los Ministros, y les confía plenamente el programa, su ejecución y su seguimiento. En el otro modelo el Presidente forma a su lado un Gabinete de expertos que reproduce, a escala reducida, el esquema de los Ministerios, y con él organiza y vigila el trabajo de los Ministros; es el esquema de la desconfianza [...]. Yo he preferido siempre el primer modelo, más próximo al modelo tradicional inglés, que hace al Presidente un primus inter pares. Felipe González ha preferido el segundo modelo, más próximo al francés».

3 Este pequeño Gabinete muestra una cierta continuidad con el que Suárez tenía como secretario general del Movimiento Nacional: Manolo Ortiz; José Manuel Otero Novas; Aurelio Delgado, «Lito», el cuñado del mismo Suárez, que dirigía su secretaría particular; un secretario de despacho, Aurelio Sánchez-Tadeo; y dos secretarias, Julia Martínez Lafuente y Ana Martínez de Leiva.

4 Entrevista con Manuel Ortiz, subsecretario de despacho (1976-1977) y secretario de Estado para la Información y portavoz del Gobierno del presidente Suárez (1978-1979). 
Gabinete, el diplomático Alberto Aza, y la incorporación de un portavoz del Gobierno, Fernando Ónega, y, posteriormente, los secretarios de Estado de Comunicación, Josep Meliá y Rosa Posada. El Gabinete estaba constituido por una estructura mínima, con una Secretaría General, una Dirección de Estudios y una Dirección de Coordinación, con rango de Dirección Gene$\mathrm{ral}^{5}$. De esta forma, el asesoramiento que recibía el presidente se ceñía al ámbito internacional, con el equipo diplomático formado por Alberto Aza, Eugenio Bregolat, Inocencio Arias y José Coderch, y al área económica, a través de Alberto Recarte, aunque también desarrolló algunas iniciativas políticas propias de gran calado, como la creación de la Comisión de Subsecretarios o el proyecto preliminar de la Ley para la Reforma Política que se entregó, siguiendo instrucciones del propio Suárez, a Torcuato Fernández Miranda para su redacción definitiva; asimismo, algunos de sus miembros desarrollaron reuniones de trabajo con miembros de la Ponencia constitucional de UCD, especialmente para la formulación de la estructura de la Constitución ${ }^{6}$.

La misma pauta de austeridad se mantuvo con el Gabinete de Calvo-Sotelo, asistido por su jefe de Gabinete, Eugenio Galdón, y un equipo reducido de colaboradores directos (Calvo-Sotelo, 1990: 175-177): Luis Sánchez Merlo, en la Secretaría General de la Presidencia; Matías Rodríguez Inciarte, primero, encargado de la preparación del Consejo de Ministros y de la relación con los ministerios y, después, ministro de la Presidencia; el portavoz del Gobierno, Ignacio Aguirre; Antonio Fournier, diplomático y asesor en materia de política exterior; Alfredo Sánchez Bella, abogado del Estado, que preparaba los dictámenes jurídicos para el presidente; y Lucila Martín, su secretaria particular?.

5 Real Decreto 2158/1978, de 1 de septiembre, por el que se estructura el Gabinete del presidente del Gobierno, art. 3. En esta inicial formulación aún no se especifican ni las funciones ni las competencias del Gabinete.

6 Entrevista con Manuel Ortiz. Según Ortiz, el proyecto original de la LRP fue elaborado por el propio Ortiz y por uno de sus colaboradores, Luis Cortés Durán, en el Gabinete del presidente y entregado a Fernández Miranda, quien lo modificó y le dio su forma definitiva antes de que el mismo Suárez lo trasladara a un grupo de trabajo, formado por diversos ministros. Luis Enrique Cortés Durán había sido director del Gabinete del ministro secretario general del Movimiento, y previamente fue director del Gabinete Técnico de la vicesecretaría general del Movimiento (BOE núm. 41/1976, de 17 de febrero de 1976).

7 Real Decreto 325/1981, de 6 de marzo, por el que se reestructuran determinados órganos de la Administración del Estado. 
Cuando UCD abandona el poder en 1982, los órganos de apoyo al presidente consistían en un secretario de Estado adjunto al Presidente, la Secretaría General de Presidencia, la Secretaría de Estado para la Información, y tres departamentos, con carácter de Dirección General: la Secretaría General de Presidencia, el Gabinete Técnico y la Dirección de Estudios. A ellos hay que ańadir tanto el vicepresidente, asistido por una unidad con rango de Subsecretaría y otra de Dirección General, y el ministro adjunto al presidente, del que dependía una subsecretaría. A estas escasas unidades habría que sumar las que dependían del Ministerio de la Presidencia, como la Secretaría de Estado para las Relaciones con las Cortes y los órganos encargados de la la función pública.

El crecimiento de este reducido Gabinete para aproximarse a los clásicos modelos europeos, en base a la necesidad de desarrollar un control más estricto sobre las decisiones de los distintos ministerios, de acuerdo a los lineamientos ya señalados de la teoría de agencia o de la delegación, se agudizó con la alternancia en el Gobierno en $1982^{8}$. Con este fin, el posterior jefe del Gabinete del presidente González, Roberto Dorado, junto a Francisco Ramos, se desplazó a Francia y a Alemania para estudiar el funcionamiento práctico de estos gabinetes y diseñar una estructura más compleja9 ${ }^{9}$. Como consecuencia de la evaluación de otras experiencias, Dorado y Ramos elaboraron un documento esencial para la transformación del modelo de Gabinete de la Presidencia del Gobierno, que suponía una mezcla entre el modelo alemán, de carácter más funcionarial, y el modelo francés, con un acento más político ${ }^{10}$. Este documento justifica, en los estrictos términos de la teoría de la delegación, la necesidad de ampliar el ámbito de actuación de los órganos de asesoramiento que rodean al presidente (Gabinete de la Presidencia del Gobierno, s. f. [1982]: 9):

En la mayoría de los países además de la clásica división entre el poder representativo (Jefatura del Estado) y el poder ejecutivo (Presidencia del Gobierno), este último se desdobla en dos o más centros neurálgicos para hacer frente a esa necesidad de dar respuestas concretas al flujo incesante de problemas que se

8 Luis Ortega (1991: 201), director del Departamento de Asuntos Institucionales del Gabinete de la Presidencia del Gobierno entre 1983 y 1987, ha señalado que el crecimiento del Gabinete en España «se homologa con los sistemas de organización presidencial más comunes en las democracias occidentales», desmintiendo la tesis del ex presidente Calvo Sotelo citada de que «el Gabinete de Felipe González está basado en la desconfianza».

9 Entrevistas con Roberto Dorado, jefe de Gabinete del presidente González (19821993), y con Francisco Ramos Fernández-Torrecilla, secretario de Estado para la Administración Pública en el primer Gobierno González.

10 Entrevista con Francisco Ramos Fernández-Torrecilla. 
presentan. Este desdoblamiento se produce inevitablemente por la fuerza de los hechos, y si no se prevé y se estructura con antelación y con racionalidad, puede dar lugar a una difuminación del poder que conduce en el límite a la irresponsabilidad dentro del gobierno y, en el mejor de los casos, a unos mecanismos de toma de decisión complicadísimos y lentísimos.

Esta extensión de las funciones y competencias del Gabinete, con la llegada del PSOE al Gobierno, a la que hemos hecho referencia, siguiendo el modelo francés, implicaba la superposición de una estructura «bicéfala» o «bipolar» o de «bipolaridad operativa», como indica el documento de trabajo referido, que "permitiría al presidente elevar al máximo el papel que le asigna la Constitución, elevándose y proyectando una imagen de hombre de estado», semejante a la distribución de roles o funciones del sistema político de la $\mathrm{V}$ República entre el presidente y el primer Ministro ${ }^{11}$. Esta bicefalia no solo es defendida en virtud de evitar la "difuminación del poder» y la irresponsabilidad ministerial o con el propósito de agilizar los procedimientos de toma de decisiones, sino para evitar, asimismo, ciertas resistencias burocráticas frente al programa del nuevo Gobierno (ibid., 10):

Si en condiciones normales parece recomendable una estructura bicéfala al estilo francés del poder ejecutivo máximo, en las circunstancias previsibles en las que podría desenvolverse un gobierno socialista en España (resistencias activas y pasivas de ciertos sectores de la Administración, y de los poderes fácticos, inexperiencia y falta de rodaje del equipo de gobierno, demandas sociopolíticas urgentes que precisarán una respuesta inmediata y coordinada si no queremos defraudar rápidamente a los que nos eligieron) parece hacer imprescindible adoptarlo para evitar una dispersión de esfuerzos, una descoordinación, una posible guerra subterránea entre los Ministerios para ampliar su esfera de competencias y sobre todo, para eliminar la tentación de crear minipoderes autónomos, insolidarios y centrífugos en el interior del gobierno.

Este cambio de modelo organizativo también tuvo su reflejo en la cúspide del Gabinete, ya que, a partir de este momento, se produjo un cambio en

11 En el mismo documento de trabajo se explica que «aunque constitucionalmente el sistema francés y el español son diferentes, la solución de reparto de funciones que existe en el primero parece haber demostrado, por una parte, su operatividad ejecutiva y por otra, su utilidad para preservar a la cúspide del ejecutivo de la erosión de lo cotidiano y para reservarle la capacidad de reflexión necesaria que exige la alta dirección, lo cual nos inclina a recomendar su adaptación práctica en un eventual ascenso socialista al gobierno» (ibid., 9). 
el perfil de los jefes de Gabinete. Suárez y Calvo-Sotelo habían optado por un modelo más «funcionarial» y por un tipo de Gabinete más próximo a la funcionarizada Oficina del Primer Ministro inglés: Alberto Aza era un diplomático de carrera, que había sido subdirector de la Oficina de Información Diplomática; Eugenio Galdón era un economista del Estado y había ocupado previamente diversos cargos en la Administración pública española. En cambio, los directores de Gabinete posteriores han tenido un perfil político y su procedencia ha sido externa a la Administración pública: Roberto Dorado era un militante del PSOE; Carlos Aragonés era un militante del Partido Popular, asesor de Aznar en su etapa como presidente autonómico; José Enrique Serrano era un abogado vinculado al PSOE, etc. Una transformación análoga se produjo, en el propio modelo inglés, con Blair de premier a finales de los ańos noventa ${ }^{12}$.

Por último, el papel que iba a ejercer en 1982 el nuevo vicepresidente del Gobierno, Alfonso Guerra, obligaba a generar una estructura organizativa en la que «la vicepresidencia ejercería de superjefe del Gabinete», hecho que se justificaba a partir de ciertas experiencias previas análogas en la historia reciente española (como la dualidad Franco-Carrero o Suárez-Abril) y sobre la base del reparto de funciones en el seno del PSOE, ya que "este modelo se ajusta al que ya existe en el interior del partido, por lo que sería visto como natural el que se reprodujese el mismo reparto de funciones en el Ejecutivo presidido por los socialistas, tanto desde la perspectiva interna, como desde la externa».

Esta situación cambió bajo la presidencia de José María Aznar, que, de entrada, nombró a varios vicepresidentes, lo que tuvo consecuencias directas sobre el poder y la capacidad de influencia de los jefes de Gabinete del Presidente. Una mayor autonomía y poder del jefe del Gabinete, sin duda, se

12 El secretario del Gabinete dirige el Cabinet Office y supone el cargo de mayor relieve del Civil Service británico, y suele ser desempeńado durante largos períodos de tiempo por el mismo funcionario (Santolaya, 1991: 99-101). Por ello, el intento de Blair de introducir un jefe de Gabinete y ampliar su staff asesor, siguiendo los clásicos modelos continentales, generó numerosos conflictos. Como ha señalado el propio Blair (2010: 16-19), «el sistema británico es gestionado básicamente por funcionarios de carrera hasta los niveles más altos. Los asesores especiales son pocos, y no muy frecuentes, a diferencia del sistema estadounidense, por ejemplo, que tiene miles. Cuando, tras unos años en el gobierno, acumulé setenta de ellos, a algunos les pareció un pequeño escándalo constitucional [...] Yo no podía creer, y sigo sin creerlo, que mis predecesores no tuvieran un jefe de Gabinete de facto, pero Jonathan [Powell] fue el primero que fue reconocido públicamente y nombrado para el cargo...». Una impresión coincidente ha expuesto el propio Powell (2011: 80) en sus memorias de los años en Downing Street. 
comenzó a desarrollar a partir del momento en que dejó de estar subordinado a los vicepresidentes de turno y solo respondía ante el mismo presidente. De hecho, los vicepresidentes posteriores ya disponían, de hecho, de un pequeño staff de asesoramiento propio y de su propio jefe o jefa de Gabinete: Francisco Villar, con Rajoy; Ignacio Sánchez Illera, con Fernández de la Vega; Gregorio Martínez Garrido, con Rubalcaba; María González Picó, con Sáenz de Santamaría, etc.

El crecimiento experimentado por el Gabinete a partir de la implantación de este modelo le fue aproximando a sus homólogos europeos en número de efectivos. A mediados de los años noventa, estos oscilaban desde el medio millar de miembros, en Alemania, hasta menos de veinte asesores, en los pequeños gabinetes de Austria, Noruega, Dinamarca o Irlanda, ocupando Francia una posición intermedia (Müller-Rommel, 1993: 133-134; Müller-Rommel, 2008). Esta expansión ha sido similar en casi todos los casos: por mencionar un solo ejemplo, el Bundeskanzleramt o Cancillería alemana, que estaba compuesta, por unos 120 asesores en la etapa de Adenauer, creció hasta alcanzar el medio millar a finales de los ańos noventa y situarse en alrededor de 615 miembros en 2015 (Kölling, 2017: 8).

En España, a comienzos del mandato de José María Aznar como presidente, aproximadamente millar y medio de empleados trabajaban en la Presidencia del Gobierno, de los cuales el Gabinete y el entorno del presidente, en sentido estricto, concentraban unos 550 efectivos y el Ministerio de la Presidencia, unos 900 (de ellos, la tercera parte como adscritos a la Oficina del Portavoz), siendo unos 767 los funcionarios de carrera, 517 el personal no funcionario y 159 los puestos de confianza (Molina, 1998: 21). En 2014 el número de funcionarios de Presidencia ascendía a más de un millar, repartidos del siguiente modo: aproximadamente, 430 en Presidencia del Gobierno, en el Gabinete y el entorno del presidente, y alrededor de 600 en el Ministerio de la Presidencia ${ }^{13}$.

13 En el diseño inicial del Gabinete en 1982, Roberto Dorado propuso elevar el rango del jefe de Gabinete a la categoría de secretario de Estado. De hecho, en la actualidad este es el rango formal del director del Gabinete. La propuesta inicial implicaba que el subdirector del Gabinete tendría nivel de subsecretario y los directores de Departamento y secretario del Gabinete, de directores generales; en correspondencia, los asesores ejecutivos tendrían el rango de subdirectores generales y sus subordinados, los asesores adjuntos, la de jefes de servicio. Sin embargo, en el proceso de transición de Gobierno se produjo una maniobra interna coordinada entre el secretario del vicepresidente, Rafael Delgado, el portavoz del Gobierno, Eduardo Sotillos, y el secretario general de la presidencia, Julio Feo, para evitar ser homologados a los subsecretarios (Feo, 1993: 209). Dorado manejaba la opción alternativa de mantener 
Hay que destacar, como elemento relevante, que de estos datos solo los que ocupan posiciones 28,29 y 30 , como funcionarios o asimilados, son los que conforman el núcleo del Gabinete del presidente, siendo el resto puestos de apoyo. Así, a principios de los años noventa el Gabinete estaba compuesto por 39 vocales asesores, con un nivel 30 de complemento de destino, y por 31 consejeros técnicos, con el nivel 28, lo que suponía un total de unos 70 asesores para los 8 departamentos del Gabinete del presidente González (Ortega, 1991: 229) ${ }^{14}$. Según los datos de Molina (1998: 17), a finales de 1997 los primeros ascendían a 23 y los segundos solo a 10 . Sin embargo, no había variado sustancialmente «el tamańo del personal funcionario, no funcionario o de confianza [...] ya que las reducciones en el número de miembros del Gabinete del Presidente se [habían] compensado con la creación de la Oficina del Presupuesto y el refuerzo de otras unidades de asesoramiento en el Ministerio de la Presidencia» (Molina, 1998: 38). En promedio, durante el gobierno de Rajoy, el número de vocales asesores había aumentado hasta, aproximadamente, 57, y el de consejeros técnicos se había estabilizado en alrededor de 30, lo que muestra que el personal al servicio del Gabinete no ha sufrido grandes cambios desde entonces, aunque el número de miembros del Gabinete ha experimentado un ligero incremento (véase la Tabla 1 del anexo para el reparto por grados y niveles funcionariales en 2014).

Esta tendencia es acorde con el crecimiento de los gabinetes en todas las democracias de nuestro entorno: en Canadá, un país solo ligeramente menos poblado que España, la Oficina del Primer Ministro contaba con más de un millar de miembros de los que 125 podían considerarse como asesores de confianza; en Australia, con una población sensiblemente inferior, el número de miembros de la Oficina del Primer Ministro era de 550, de los que solo alrededor de 50 podían considerarse como asesores directos —estos últimos se

para su cargo de jefe de Gabinete el rango de subsecretario y el resto de puestos mencionados el de directores generales. Finalmente, lograron impedir los planes de Dorado, siendo el portavoz del Gobierno el único secretario de Estado y los demás, incluido el director del Gabinete, subsecretarios. El subdirector y el secretario del Gabinete adquirieron, inicialmente, los rangos de director general y subdirector general, respectivamente. Solo, años después, el director asumió el nivel de secretario de Estado y el subdirector, el de subsecretario.

14 Entrevista con Roberto Dorado. Según Dorado, pese a que habían calculado una estructura de alrededor de 120 personas, casi nunca superaron los 60 miembros. La razón para la que no creciera más el Gabinete durante la primera etapa de gobierno socialista era doble: en primer lugar, por un problema de infraestructuras y espacios en el complejo de la Moncloa y, en segundo lugar, porque no encontraron el personal necesario y carecían de suficientes asesores de calidad vinculados al partido. 
habían incrementado desde 30 a comienzos de los años noventa hasta los 53 de finales de 2012 - (Truswell y Atkinson, 2011: 10; Rhodes y Tiernan, 2014b:7), etc.

\section{PODER DURO VS. PODER BLANDO: ESTRUCTURA, COMPETENCIAS Y FUNCIONES DEL GABINETE}

\section{LOS NIVELES DE LA FORMALIDAD}

El Gabinete español no es, como el staff de la Casa Blanca, un gabinete de carácter ejecutivo, que impone sus criterios en nombre del presidente (hard power $)^{15}$. En España no actúa por propia iniciativa y, en este sentido, desarrolla un cierto poder blando (soft power), similar al de los gabinetes de los primeros ministros de los sistemas parlamentarios europeos. De ahí que, de acuerdo a las encuestas realizadas a los ministros, hace unos años, acerca de su percepción sobre las funciones más importantes desarrolladas por los staffs de los primeros ministros europeos, más del $90 \%$ de ellos clasificó la función de asesoría al primer ministro como la más importante de los gabinetes, seguida por la preparación de la agenda del gobierno (72\%), el control de propuestas de los ministerios ( $43 \%$ ) y, en último lugar, la de poner en marcha sus propias propuestas (36\%) (Müller-Rommel, 1993: 137) ${ }^{16}$.

Aunque estas competencias, y su importancia en el trabajo diario del Gabinete, puedan estimarse cuantitativamente, hay que subrayar que no son estáticas, sino que presentan una evolución dinámica y, en la práctica, tienden a prevalecer unas u otras en función del perfil del presidente y de su estilo de gobernar. En España, de hecho, los gabinetes socialistas bajo Felipe González tendieron más al control de la actividad ministerial, debido al papel del vicepresidente en la dirección del Gabinete. En el mismo documento elaborado por los dirigentes socialistas para la transformación del modelo de Gabinete de la Presidencia del Gobierno en 1982, ya sobresale, al margen de esas competencias formales recogidas en el Real Decreto de organización del Gabinete,

15 Entrevista con Roberto Dorado.

16 Esta jerarquía de funciones es distinta según las investigaciones realizadas para las nuevas democracias de la Europa Oriental (Müller Rommel, 2008). En estos países, las funciones del staff del primer ministro con más impacto son la preparación de la agenda del gobierno (59\%), seguida por el asesoramiento al primer ministro (27\%), el control de las propuestas ministeriales (10\%) y el desarrollo de sus propias propuestas políticas $(4 \%)$. 
este aspecto. Entre los criterios organizativos básicos se subraya el «papel director del Gabinete de Presidencia sobre los demás Gabinetes y el papel de coordinador que debe ejercer éste sobre los titulares de los ministerios", ya que «debe cubrir el estricto seguimiento de todos los Departamentos Ministeriales y sus actuaciones» (Gabinete de la Presidencia del Gobierno, s.f. [1982]: 4, 6). Se concibe al Gabinete como un "estado mayor» o "centro neurálgico» del Gobierno, encargado de controlar la acción de este. En segundo lugar, se pone de relieve la tarea de velar por el cumplimiento del programa electoral del partido, competencia que deriva de su composición estrictamente política, puesto que debe de estar «formado por personas del partido o muy afines al mismo que tuvieran además un conocimiento mínimo del funcionamiento de la maquinaria administrativa» (Gabinete de la Presidencia del Gobierno, s.f. [1982]: 7). En la etapa de Aznar, en cambio, predominó la tradicional función de asesoría al primer ministro, de «apoyo material al presidente, su correspondencia, su agenda, su discurso... y en una forma mucho menos intensa como un instrumento de recepción y transmisión de información alternativa» ${ }^{17}$.

Las funciones específicas y singulares del Gabinete de la Presidencia del Gobierno fueron establecidas, de una manera clara, a partir de la instauración del modelo continental en España con la llegada del presidente González a Moncloa en 1982, siendo ordenadas alrededor de cuatro ejes de actuación, que no han sido modificados en regulaciones posteriores ${ }^{18}$. En primer lugar, proporcionar, al presidente y al vicepresidente, toda la información necesaria para el correcto ejercicio de sus funciones. En segundo lugar, asesorar en todos los asuntos que sean requeridos por ambos cargos. En tercer lugar, facilitar al presidente la coordinación de la acción gubernamental a partir del análisis de los planes de acción de los distintos departamentos ministeriales. Por último, como una cláusula general, realizar todas las actividades encomendadas por el presidente.

Esto significa que de las distintas funciones políticas (asesorar al primer ministro y desarrollar propuesta políticas propias) y administrativas (preparar la agenda del Gobierno y controlar las propuestas de los ministros) de los gabinetes, que ha estudiado comparativamente Müller-Rommel (1993 y 2008), el español se concentra, incluso desde una perspectiva formal, en las mismas que el resto de los gabinetes de los primeros ministros: en el asesoramiento y seguimiento de las actividades ministeriales, sin dejar de lado el impulso de propuestas, una función de más relieve durante la etapa de Aznar, pero también presente

17 Entrevista con Gabriel Elorriaga, subdirector del Gabinete del presidente Aznar (1996-2000).

18 Real Decreto 3773/1982, de 22 de diciembre, por el que se determina la estructura orgánica de la Presidencia del Gobierno. 
en el extenso período de mandato de González ${ }^{19}$. Estas iniciativas, en ocasiones, son estrategias amplias que desembocan en planes conjuntos de varios ministerios que tienen su origen en propuestas del propio Gabinete, que es quien articula paquetes de reformas que incluyen un conjunto heterogéneo de actuaciones que, por su naturaleza, difícilmente podrían surgir en el seno de un único departamento ministerial. Un buen ejemplo de este último tipo de propuestas o iniciativas fue el Plan de Seguridad Ciudadana de 2002, que incluía una batería de medidas contra la delincuencia que abarcaban acciones combinadas de política judicial y procesal, de reforma del Código Penal y de la Ley de Procedimiento Criminal y de la política penitenciaria, ámbitos correspondientes a distintos ministerios como Justicia o Interior, etc. ${ }^{20}$.

La principal función del Gabinete en términos formales se extiende a la constante presencia de sus miembros en los órganos colegiados. Esta función de control se revela como un eficaz mecanismo ex post de control e información acerca de las actividades de sus agentes por parte del presidente. Puesto que el Gabinete es un órgano de "coordinación de la información política» y no un «órgano de coordinación administrativa», indudablemente «es fundamental su presencia en los órganos colegiados que desarrollan funciones generales de coordinación, como las Comisiones Delegadas del Gobierno y la Comisión de Secretarios de Estado y Subsecretarios, y funciones de coordinación más concretas, como las distintas Comisiones Interministeriales» (Ortega, 1991: 240); en este caso, el Gabinete ha de «servirle al presidente como ojos y oídos»" ${ }^{21}$. Como señala Gabriel Elorriaga, «en todos los órganos colegiados del Gobierno siempre había un miembro del Gabinete, que es lo que le permite al final al Gabinete informarle al presidente del Gobierno de qué es lo que está pasando en la Comisión Delegada de Asuntos Económicos o en la Comisión Delegada de Asuntos Culturales o en la Comisión de Subsecretarios, en la Interministerial de esto y aquello... en los veinticinco órganos colegiados que hay, siempre hay alguien del Gabinete... que le proporciona una información al presidente del Gobierno que, objetivamente, no tiene nadie, porque no la puede tener, porque no hay nadie que tenga oídos en todo el conjunto. Y eso le permite al presidente dirigir la actividad del Gobierno y coordinarla, orientarla y priorizarla ${ }^{22}$. Esta

19 Entrevistas con José María Aznar, presidente del Gobierno, y con Roberto Dorado. Según Roberto Dorado, las dos funciones principales del Gabinete de González eran, por este orden, el seguimiento de las políticas de los ministerios, el seguimiento sobre el programa electoral del partido y, en tercer lugar, hacer propuestas.

20 Entrevista con Javier Fernández Lasquetty, subdirector del Gabinete del presidente Aznar (2002-2004).

21 Entrevista con Javier Fernández Lasquetty.

22 Entrevista con Gabriel Elorriaga. 
presencia está plenamente institucionalizada y, en el caso del Gobierno de Zapatero, incluso se extendió al ámbito del seguimiento y gestión de crisis, ya que tras la etapa del PP en la que se adscribió a la Secretaría General de Presidencia, bajo la dirección de Zarzalejos, Zapatero, manteniendo dicha adscripción orgánica, decidió que, funcionalmente (en sus actuaciones, programa de trabajo, actividades, etc.), pasara a depender del director del Gabinete ${ }^{23}$.

Es esta, por tanto, una de las tareas principales del Gabinete: la función de control de la actuación de los ministerios y de vigilancia en el cumplimiento del programa electoral, de acuerdo a este planteamiento de «contrapoder» y de agente que fiscaliza la labor del otro agente del presidente, que es el Gobierno y, especialmente, los ministerios ${ }^{24}$. En el documento anteriormente reseñado de 1982 (Gabinete de la Presidencia del Gobierno, s.f. [1982]: 20-21) esta función está especialmente reflejada:

Los Departamentos deben revisar y emitir informes sobre todos los proyectos o acciones que realicen los diferentes ministerios. Al mismo tiempo deben cuidar que estos se ajusten al programa sectorial correspondiente, que se coordine con otras posibles áreas afectadas. Su labor fundamental debe ser de estímulo y colaboración hacia sus corresponsales, pero si opina en contra de lo propuesto debe intentar que modifiquen la propuesta. Si no se consigue, transmitir al nivel superior acompañando su opinión fundamentada para que se dirima en ese escalón. Esto exige que los integrantes de los Departamentos tengan una preparación técnica de primera categoría y un nivel político notable (especialmente el Director del Departamento).

La asesoría al presidente se concreta, específicamente, en proporcionarle información alternativa (no necesariamente "contrapuesta» sino, en la mayor parte de las ocasiones, "complementaria», como afirman Aragonés y Fernández Lasquetty) a la que le es suministrada desde los ministerios, con el objeto de que se forme un criterio propio ${ }^{25}$. Ello genera una «relación absolutamente

23 Entrevista con José Enrique Serrano, director del Gabinete de los presidentes González (1995-1996) y Zapatero (2004-2011).

24 Entrevista con Roberto Dorado. Dorado, por ejemplo, expresó durante la entrevista con los autores su malestar por algunas informaciones publicadas por el diario El País en las que se insinuaba que "puenteaba" a los ministerios.

25 Entrevistas con Carlos Aragonés, director del Gabinete del presidente Aznar (19962004), y con Javier Fernández Lasquetty. Según Lasquetty, los ministros «ven con desconfianza al Gabinete del Presidente porque es alguien que trabaja sobre los mismos temas, que no tiene autoridad propia, pero que está muy cerca de la persona que tiene autoridad sobre ellos mismos». 
anómala», muy similar a la que existe entre los gabinetes de los ministros y los secretarios de Estado en el nivel ministerial ${ }^{26}$. Hay que matizar que esta labor de control y/o tutela sobre los ministros no es igual para todos ellos. Es para los ministros que carecen de una base autónoma de poder o de una base de relación propia con el presidente, sobre los que el Gabinete del presidente ejerce una mayor tutela. En el caso de aquellos que tienen una base autónoma de poder, esta tutela puede generar conflictos, como muestra el caso de las continuas discrepancias entre la Oficina Económica de la Presidencia, dirigida por Miguel Sebastián, y el Ministerio de Economía, en la segunda etapa de Pedro Solbes, en un amplio espectro de temas, desde la reforma del IRPF a la actitud gubernamental ante distintas OPA, como las de Sacyr sobre BBVA o de Gas Natural sobre Endesa ${ }^{27}$.

La otra vertiente de esta labor de control es la necesidad de coordinación, puesto que, como subrayó José Enrique Serrano, tanto en el período de González como en el de Rodríguez Zapatero, «no es un problema de desconfianza, sino de contraste y de valorar no solamente algo qué significa en un área de gobierno, sino en la acción conjunta de gobierno, que eso es también una gran virtud del Gabinete» ${ }^{28}$. El propio José María Aznar subrayó también la importancia de esta función, argumentando que «la acción de gobierno no era una

26 Entrevista con Javier Fernández Lasquetty.

27 La relación entre el Gabinete y los ministros es el aspecto esencial tratado en el documento inédito «¿Qué Gabinete queremos?»: «La relación del Gabinete con los ministerios es una cuestión fundamental. La asistencia, el apoyo y el asesoramiento que el mismo puede ofrecer al presidente y al vicepresidente serán necesariamente de baja calidad si no se logra establecer una relación adecuada y fructífera con los distintos departamentos ministeriales». En el mismo texto se describe la utilidad del Gabinete para los ministerios, a los que puede ofrecer «capacidad para promover y empujar políticamente la ejecución de sus iniciativas, para mediar en los conflictos interministeriales y para allanar dificultades con la presidencia, para transmitir la línea política del Gobierno, tanto en lo que afecte a sus iniciativas como con carácter más general, para coordinar actuaciones interministeriales y para, en algunos casos, asistirlos técnicamente», (Gabinete de la Presidencia del Gobierno, s.f. [1993]: 9-10).

28 Entrevista con José Enrique Serrano. Serrano subrayó su negativa a que hubiera un asesor que hiciera un seguimiento específico a cada ministerio argumentando que él «nunca había concebido el Gabinete de la Presidencia como, por utilizar una expresión muy vieja, un comisariado político», puesto que, a su juicio, «el Gabinete es otra cosa: aquí no estamos para controlar o vigilar a cada uno de los Ministerios o a cada uno de los ministros, sino para contribuir a que el Gobierno se desempeñe como tal, como un Gobierno y no como una mera yuxtaposición de ministros». 
acción de ministerios aislados sino una acción en conjunto», una perspectiva que tiende a desarrollarse desde el Gabinete, que vela para que esa coordinación y solidaridad de la actuación gubernamental sea efectiva ${ }^{29}$. Esto exige también una enorme habilidad y una gran experiencia político-administrativa por parte del director del Gabinete, dos cualidades básicas en su perfil que se han ido acentuando progresivamente y que también son apuntadas en los estudios comparados sobre los directores de gabinetes (Rhodes y Tiernan, $2014 a$ y $2014 b)^{30}$.

\section{EL PODER BLANDO DEL GABINETE Y LA FLUIDEZ DE LA INFORMALIDAD}

Entre las funciones informales más importantes del Gabinete, que suponen el mejor reflejo del poder blando que este ejerce, se encuentra la preparación del debate parlamentario sobre el estado de la nación y las principales intervenciones parlamentarias del presidente sobre temas europeos o de seguridad, los clásicos debates de investidura, censura, comparecencia semanal en las Cámaras, etc. Además, algunos de los departamentos, como Análisis y Estudios, suelen preparar estrategias de comunicación para distintos ministerios y elaboran estudios de opinión pública sobre la valoración de la actuación gubernamental $^{13}$. Otra función informal del Gabinete es la de gatekeeper o

29 Entrevista con José María Aznar.

30 Entrevistas con José Enrique Serrano y con Carlos Aragonés. Serrano, con carácter previo a su nombramiento como jefe de Gabinete de González, había acumulado incluso dos cargos: secretario general de la Vicepresidencia y subdirector del Gabinete, con asignación de cuatro o cinco de los departamentos que formaban el mismo. Su sucesor, Carlos Aragonés, ya había sido, anteriormente, jefe de Gabinete de Aznar en su etapa de presidente de la Comunidad de Castilla y León. Moragas había trabajado en el Departamento de Protocolo de la Presidencia del Gobierno en el primer Gobierno de Aznar y, cuando iba a formar parte del Departamento de Internacional del Gabinete, el secretario general de la Presidencia, Javier Zarzalejos, le designó como su propio jefe de Gabinete.

31 El documento «¿Qué Gabinete queremos?», elaborado en la etapa final del Gobierno de Felipe González, hace una distinción entre sus labores operativas y sus labores de análisis (Gabinete de la Presidencia del Gobierno, s.f. [1993]: 2-6) y dentro de las labores operativas subraya la importancia de «todo el trabajo destinado a preparar respuestas a la cuantiosa correspondencia que reciben el presidente y el vicepresidente, valorar la oportunidad o no de conceder audiencias solicitadas, aceptar invitaciones recibidas, preparar la documentación necesaria para recibir y hacer visitas, e informar todas las iniciativas normativas que debe ver el Consejo de Ministros o sus comisiones delegadas». 
controlador del acceso al presidente, buen ejemplo de influencia en el entorno presidencial y de soft power. Esta actuación queda bien reflejada en la Tabla 3, incluida en el documento anteriormente reseñado de 1982, que señala que «el trabajo del Presidente debe ser interrumpido lo menos posible, para ello hay que determinar quién tiene libre acceso telefónico total o quién parcial» (Gabinete de la Presidencia del Gobierno, s.f. [1982]: 27). Esta distinción del acceso total o parcial queda referida, según el mismo documento interno, a la discrecionalidad del jefe de Gabinete: «Los no reseñados, salvo casos excepcionales, no se pueden comunicar directamente con él. El acceso parcial significa que hay que decir previamente que está ocupado y que se le llamará en cuanto se pueda. Quedando a discreción del director de Gabinete si se le llama o le telefonea él previamente para enterarse del asunto».

En esta función de gatekeepers, el papel del jefe del Gabinete del presidente es primordial ${ }^{32}$. El perfil político de los jefes de Gabinete ha ido cambiando, desde el control de la agenda de los presidentes y el desempeño del rol de filtros o gatekeepers, como con Carlos Aragonés o José Enrique Serrano, hasta una expansión del papel de esta figura como plataforma de proyección internacional del actual presidente del Gobierno, en el caso de Jorge Moragas. No olvidemos que los jefes del Gabinete constituyen el eje de la Presidencia del Gobierno y han ido asumiendo un poder creciente dentro del entorno presidencial en todos los países de nuestro entorno (Rhodes y Tiernan, 2014b). ${ }^{33}$

Por último, otras funciones informales se refieren a la relación del Gabinete con el partido del Gobierno, que es muy variable en el tiempo y mucho menos estable que con los ministerios, por lo que puede decirse que las relaciones suelen ser intermitentes, aunque han sido más continuadas cuando el PSOE ha dirigido el Gobierno ${ }^{34}$. Por ejemplo, con Zapatero estaban

32 Entrevista con José Enrique Serrano. Sin embargo, este papel ha variado según el presidente, ya que el propio Serrano, según manifestó, despachaba con una menor frecuencia con González que con Zapatero.

33 Los jefes de Gabinete deben ser simultáneamente "políticamente sensibles y administrativamente competentes» (Tiernan y Pfiffner, 2014: 17). Por eso, conviene subrayar de la experiencia comparada cómo la insensibilidad de algunos directores de Gabinete ha acabado por alienar a sus propios subordinados, generar conflictos con los miembros del Parlamento y del partido o derivar en relaciones de hostilidad con la prensa, como muestran los casos de Adams con Eisenhower, Haldeman con Nixon, Regan con Reagan o Sununu con Bush. Todos ellos dimitieron «tras caer en desgracia y habiendo causado un gran daño político a sus Presidentes» (Tiernan y Pfiffner, 2014: 20).

34 Entrevistas con Carlos Aragonés y con Gabriel Elorriaga. A juicio de Aragonés, la «vinculación más estrecha entre partido y Gobierno» se corresponde más con la «personalidad del Partido Socialista». 
programadas reuniones semanales del director del Gabinete (Serrano) con la vicepresidenta del Gobierno (Fernández de la Vega), el portavoz del grupo parlamentario (Rubalcaba) y el secretario de organización del partido (Blanco) con el objetivo de mejorar la coordinación entre los cuatro pilares del ejecutivo: Gobierno, partido, grupo parlamentario y Gabinete ${ }^{35}$.

Por último, entre este tipo de funciones está la creciente influencia (soft power que en algunos casos se ha transformado en verdadero hard power) del jefe del Gabinete en el diseño, planificación y coordinación de las campañas electorales de los partidos en el Gobierno, aunque hay que precisar que la relación entre los comités electorales de los partidos y los gabinetes presidenciales es de doble dirección: los miembros de los comités electorales tienden a ocupar posiciones clave en los futuros gabinetes cuando sus partidos acceden al Gobierno, y, viceversa, desde el Gobierno participan en tareas muy destacadas en la planificación y ejecución de las campañas electorales, dado su estrecho control de la agenda del presidente, que compite como candidato a la presidencia. Ejemplo del primer modelo de interrelación entre comités de campaña y el Gabinete es el caso del PSOE al acceder al Gobierno en $1982^{36}$. El trasvase de integrantes del equipo de campańa al Gabinete reducía los costes del mantenimiento de la estructura partidaria, aunque provocó que, cuando en 1996 tuvieron que regresar a la oposición, el partido, prácticamente, quedara vaciado de asesores y técnicos.

El segundo modelo de relación entre el Gabinete y los comités de campaña de los partidos con funciones de gobierno puede observarse en el PSOE y en el PP con Felipe González y Mariano Rajoy. El comité electoral del PSOE en las campañas de elecciones generales presenta una cierta diferenciación respecto al órgano dirigente del partido y registra una acusada tendencia hacia la "profesionalización». Suele ser designado por el candidato a la presidencia del Gobierno y dirigido por un coordinador general que ha sido, tradicionalmente, el vicesecretario general del partido o el secretario de

35 Entrevista con José Enrique Serrano.

36 La gran relación entre el comité electoral del partido y el futuro Gabinete de la Presidencia bajo Felipe González queda bien reflejada en esta descripción crítica de Julio Feo (1993: 208), aludiendo a la campaña de 1982 y a los planes de los miembros del comité electoral de ocupar todos los puestos relevantes del futuro Gabinete: «El ambiente de Bravo Murillo era más hostil... pues... se habían desbaratado los planes del pequeńo aparato del comité electoral que estaba alrededor de Alfonso Guerra y que estaba compuesto principalmente por Roberto Dorado, Helga Soto, Nacho Varela, Fernando Valderrama y Javier Tezanos. Entre ellos habían hecho un esquema y un organigrama de lo que tenía que ser la Presidencia del Gobierno y parece ser que ya se habían repartido los puestos». 
organización. Durante la etapa en la que Alfonso Guerra dirigía el comité electoral del partido, Roberto Dorado desempeñaba la función de vicecoordinador electoral, duplicando una colaboración análoga a la que mantenían en el seno del Gobierno como vicepresidente y jefe del Gabinete del presidente.

En el caso del PP, el comité ejecutivo nacional, a propuesta del presidente del partido, suele designar un director de la campaña y un comité electoral, que tiene una elevada coincidencia con la directiva del partido. Precisamente, en las campañas de 2015 y 2016, el jefe del Gabinete del presidente del Gobierno, Jorge Moragas, fue designado como director de la campaña ${ }^{37}$. En un segundo nivel, el comité de campaña estaba apoyado por un equipo de unas cincuenta personas, dirigido por Alfonso de Senillosa, director adjunto del Gabinete de Presidencia del Gobierno, cuyas reuniones también solía presidir Moragas. Debido al peso político atribuido a Jorge Moragas, este equipo de campaña registró una fuerte presencia de sus colaboradores en el Gabinete de Moncloa ${ }^{38}$.

\section{LA PÉRDIDA DE AGENCIA Y EL CONTROL MINISTERIAL COMO PREMISA DE LA ESTRUCTURA ORGANIZATIVA DEL GABINETE}

Analizadas las competencias formales y las funciones informales y los diversos niveles de poder blando que ejerce el Gabinete, procede concluir con una breve aproximación a la relación entre su estructura organizativa y las tareas que tiene atribuidas este órgano en su carácter de agente encargado de reducir la pérdida de agencia del presidente con sus ministros. En el diseño original de la

37 Entrevistas con Eugenio Nasarre, Alfredo Timermans y Gabriel Elorriaga. En la campaña de 2000, el papel del Gabinete del presidente Aznar aparece más diluído, pero también fue muy relevante, estructurando uno de los dos equipos no formalizados de la campaña. Este equipo estaba formado por el director, el subdirector y por cuatro directores de los cinco departamentos en los que se divide dicho Gabinete: Aragonés, director del Gabinete y vocal del Comité Ejecutivo del PP; Elorriaga, subdirector del Gabinete; Timermans, de Asuntos Institucionales; Tomé, de Asuntos Económicos y Sociales; Gil Casares, de Internacional y Defensa; y Nasarre, de Análisis y Estudios. Si bien en estas elecciones, tanto la precampaña como la campaña electoral, aparecieron como concebidas por el PP, en realidad su diseño tuvo lugar en Moncloa.

Formaban parte José Sánchez Arce, Ignacio Peyró, Edelmira Barreira, Sergio Ramos o Abelardo Bethencourt — jefe de Gabinete de Moragas_, Valle Ordóńez —la responsable de la agenda del presidente- y distintos cargos del partido. En un tercer nivel, más especializado, constituido por equipos específicos de «unidad de mensaje», «redes sociales», «segmentación» o "comunicación», la presencia de miembros del Gabinete de Moncloa era evidente. 
estructura moderna de los actuales gabinetes de Presidencia en 1982 hubo que resolver tres problemas iniciales: en primer lugar, el debate acerca de si el Gabinete debía depender de la vicepresidencia o del Ministerio de la Presidencia; en segundo lugar, el problema del reparto o duplicidad de gabinetes entre el presidente y el vicepresidente o entre el presidente y el Ministerio de la Presidencia; y, en tercer lugar, la estructura específica por áreas sectoriales y cuál sería el número de estas. Dado el control que iba a ejercer el vicepresidente Alfonso Guerra sobre el Gabinete, se descartó la alternativa de que este desarrollara su labor bajo la dependencia del Ministerio de la Presidencia, con el argumento de que al no tener competencias específicas, la vicepresidencia, a diferencia del Ministerio de la Presidencia, podía «desempeñar su labor de coordinación y control exclusivamente». Por lo que respecta a la posible duplicidad de dos gabinetes, uno perteneciente a la Presidencia (completo o incompleto, y en este caso, con funciones especializadas en los ámbitos de Asuntos Exteriores, Mercado Común, Defensa e Interior) y otro correspondiente a la vicepresidencia o al Ministerio de la Presidencia (completo o incompleto, y en este supuesto, asumiendo el resto de las áreas de políticas públicas), se prefirió la opción de la vicepresidencia como «superjefe del Gabinete» por economía de recursos humanos, por mejor coordinación del propio Gabinete y del ámbito de la Presidencia y mejor control y planificación del Gobierno.

Finalmente, requieren una discusión más detenida las sucesivas decisiones de los diferentes presidentes en cuanto a la estructura sectorial de los distintos departamentos del Gabinete. El diseño inicial de los socialistas implicaba una distribución en siete áreas, un número intermedio entre los nueve departamentos existentes en Francia y los seis de la Cancillería alemana, los dos países que habían sido visitados por Dorado y Ramos: 1) Relaciones Institucionales, Administración Territorial y Justicia e Interior; 2) Economía; 3) Sanidad, Seguridad Social y Trabajo; 4) Asuntos Exteriores; 5) Cultura y Educación; 6) Defensa y Servicios Secretos, y 7) Planificación, Estrategia, Medios de Comunicación y Coyuntura Política. A estos siete departamentos se ańadió, posteriormente, en 1989, el de Estudios, orientado al análisis de tendencias y a los problemas a medio plazo de la política nacional, y alejado, por tanto, de la problemática cotidiana ${ }^{39}$. En 1987 se incorporó al Gabinete un área para el seguimiento de las situaciones de crisis y emergencia, nacionales e internacionales, de los planes y programas de contingencia para el mantenimiento de las infraestructuras básicas y la protección civil en este tipo de situaciones ${ }^{40}$. Esta estructura hipertrofiada tenía como finalidad construir un

39 Real Decreto, 1481/1989, de 15 de diciembre.

40 Real Decreto 163/1987, de 6 de febrero. 
Gabinete orientado, primordialmente, al control de la actividad del Gobierno, puesto que el vicepresidente Guerra no tenía bajo su responsabilidad ningún ministerio y su única función era la dirección del Gabinete ${ }^{41}$.

El presidente Aznar desarrolló una política de reducción del Gabinete, disminuyendo el número de asesores, separando los apoyos del presidente de los del vicepresidente y remodelando la estructura del Gabinete de la presidencia, limitando estas ocho áreas a cinco: 1) Asuntos Económicos y Sociales; 2) Internacional y Defensa; 3) Educación y Cultura; 4) Análisis y Estudios, y 5) Asuntos Institucionales. El segundo elemento de esta política de reducción del Gabinete fue la adscripción de servicios como la denominada línea caliente o el Departamento de Infraestructuras y Seguimiento de Situaciones de Crisis a la Secretaría General. En general, Aznar siguió el criterio de mantener «un Gabinete sustancialmente más reducido que el anterior», porque siempre tuvo «la idea de no tener gabinetes muy extensos... sino gabinetes concentrados en los temas y con personas muy seleccionadas» ${ }^{42}$. No obstante, esta política restrictiva se vio compensada, parcialmente, por la introducción de la Oficina Presupuestaria. Es reseñable que, en algunas áreas, se produjo una cierta continuidad del personal, especialmente en los Departamentos de Internacional y Defensa, donde se mantuvo «la parte más profesionalizada y menos politizada» de la estructura, pese al gran cambio experimentado en otros departamentos ${ }^{43}$.

El presidente Rodríguez Zapatero redujo, más aún, esta estructura manteniendo solo cuatro departamentos: 1) Asuntos Institucionales; 2) Política Internacional y Seguridad; 3) Análisis y Estudios, y 4) Educación y Cultura. El presidente Rajoy, en 2011, retornó a una estructura similar a la de Aznar, con cinco áreas: 1) Asuntos Internacionales; 2) Análisis y Estudios; 3) Políticas Sociales; 4) Educación, Ciencia y Cultura, y 5) Comunicación con los Ciudadanos ${ }^{44}$. Asimismo, se creó un Departamento de Seguridad Nacional, que también coordina actualmente la política de seguimiento de crisis. Por último, tras la reciente reforma de julio de 2017, se ha reforzado la asesoría al presidente en política exterior con la creación de dos departamentos distintos referidos a este ámbito: Asuntos Internacionales y Asuntos Europeos y G-20 ${ }^{45}$.

41 Entrevista con Gabriel Elorriaga.

42 Entrevista con José María Aznar. Tanto Aznar como Aragonés confirmaron que la fusión de materias o áreas fueron decisiones del presidente respecto al Gabinete.

43 Entrevista con Gabriel Elorriaga.

44 Real Decreto 94/2015, de 13 de febrero (BOE del 14 de febrero), modificando el inicial Real Decreto 83/2012.

45 Real Decreto 766/2017, de 28 de julio (BOE del 29 de julio), que modifica lo dispuesto por el Real Decreto 426/2016, de 11 de noviembre (BOE del 12 de noviembre). 
Comparativamente, no hay demasiadas diferencias, desde el punto de vista de su estructura organizativa, con otros gabinetes, como ya se señaló al mencionar los casos alemán y francés. Un buen ejemplo es la Cancillería alemana, que cuenta con departamentos de política social, medio ambiente, política económica y fiscal, asuntos europeos, política internacional e inteligencia y seguridad nacional. Otra buena ilustración es el caso australiano, cuyo Gabinete dispone de similares departamentos de política doméstica, política internacional y de seguridad nacional, política estratégica e implementación y gobernanza.

Mención aparte merecerían los distintos órganos externos al Gabinete que han compartido funciones de apoyo a la Presidencia del Gobierno: la Secretaría General de Presidencia, el Ministerio u Oficina del Portavoz del Gobierno, el Ministerio de Relaciones con las Cortes o el Ministerio de la Presidencia, que requerirían de un análisis específico, ajeno al objeto de estudio principal de este trabajo (Paniagua, 2017) ${ }^{46}$. No obstante, algunas de estas funciones, como las de la Secretaría General de Presidencia, durante el mandato de Rajoy han sido absorbidas paulatinamente por el Gabinete, permitiendo ampliar sus competencias.

\section{CONSIDERACIONES FINALES}

El primero de los objetivos de esta investigación ha sido presentar un análisis no formalista y político del funcionamiento práctico del Gabinete de la Presidencia del Gobierno a partir, por vez primera, de las entrevistas en profundidad realizadas a los distintos directores del Gabinete que han desempeñado este cargo desde la transición de la democracia a la actualidad y también a uno de los presidentes del Gobierno en ejercicio a lo largo de este extenso periodo. Asimismo, este trabajo ha revelado algunos aspectos desconocidos de la conformación del moderno "complejo de la Moncloa» a partir de determinados documentos inéditos que explican la concepción y génesis del moderno Gabinete español, en base a algunos modelos europeos.

Las conclusiones principales que se desprenden de este análisis, contrastado con sus principales protagonistas, son, en primer lugar, que el Gabinete

46 No hay que olvidar, tampoco, diversas funciones complementarias que debe cumplir este órgano de apoyo al presidente del Gobierno, que solo aparecieron recogidas en la nueva regulación del Gabinete con Mariano Rajoy en 2011 (Real Decreto 83/2012, de 13 de enero, de reestructuración de la Presidencia del Gobierno): a) Organización de eventos y seguridad; b) Protocolo; c) Recursos humanos y medios materiales; d) Logística de desplazamientos y viajes e) Salud y Sistema Operativo Sanitario de la Presidencia; f) Cláusula residual: incluye la ejecución de todas aquellas otras actividades o funciones que le encomiende al Gabinete el presidente del Gobierno. 
es un órgano técnico y político de asesoramiento del primer ministro que actúa como un agente capaz de dotar al presidente de información propia, al margen de la que puede obtener de los ministros que componen su Gobierno, por lo que actúa como un segundo agente que permite reducir ex post las pérdidas de agencia, de acuerdo con lo establecido por la teoría de la delegación, que todo proceso de atribución de poder a distintos órganos genera. En segundo lugar, ha permitido conceptualizar el Gabinete español como un órgano que dispone no solo de una capacidad de iniciativa política propia, sino también de un cierto poder blando (soft power) o poder de influencia, que lo asemeja a sus homólogos europeos, cuyos modelos reprodujo en su génesis, pero, a su vez, lo diferencia de otros gabinetes, como el norteamericano, que dispone, efectivamente, de una capacidad ejecutiva propia. En tercer lugar, no solo se han descrito en la práctica las funciones formales desarrolladas por los gabinetes en la Moncloa, desde el inicio de la democracia, y ya analizadas en algún estudio jurídico previo, sino que, además, se han puesto de relieve las numerosas, y crecientes, competencias informales del Gabinete en la gestión ordinaria de los asuntos políticos, en aspectos que van desde su participación en la preparación de intervenciones presidenciales o en comisiones de seguimiento de crisis a su presencia cada vez más activa en campańas electorales. Finalmente, se han analizado otros aspectos relevantes como las relaciones, no siempre pacíficas, del Gabinete con actores importantes en el proceso de decision-making, como los ministros, el partido y el grupo parlamentario que sostiene al Gobierno, y se ha bosquejado un perfil de los jefes de Gabinete que han desempeñado el cargo a lo largo de estos años.

\section{Bibliografía}

Blair, T. (2010). A Journey. Londres: Hutchinson.

Burch, M. y Halliday, I. (2004). The Blair Government and the Core Executive. Government and Opposition, 39 (1), 1-21. Disponible en: https://doi.org/10.1111/j.0017257x.2004.00029.x.

Calvo-Sotelo, L. (1990). Memoria viva de la transición. Barcelona: Plaza \& Janés.

Camerlo, M. (2014). La centralidad del centro: rol y relevancia de las Secretarías Presidenciales en Argentina. Ponencia presentada al Seminario Centro Presidencial. América Latina, Estados Unidos, España. Montevideo: Instituto de Ciencia Política, Universidad de la República.

Cohen, D. B., Hult, K. M. y Walcott, C. E. (2012). The Chicago Clan: The Chiefs of Staff in the Obama White House. Social Science Quarterly, 93 (5), 1101-1126. Disponible en: https://doi.org/10.1111/j.1540-6237.2012.00919.x.

Elgie, R. (2011). Core Executive Studies Two Decades on. Public Administration, 89 (1), 64-77. Disponible en: https://doi.org/10.1111/j.1467-9299.2011.01899.x.

Feo, J. (1993). Aquellos años. Barcelona: Ediciones B. 
Gabinete de la Presidencia del Gobierno (s.f. [1982]). Estudio-Propuesta de la estructura y funciones de la Presidencia del Gobierno [informe inédito]. Madrid: Presidencia del Gobierno.

Gabinete de la Presidencia del Gobierno (s.f. [1993]). ¿Qué gabinete queremos? [informe inédito]. Madrid: Presidencia del Gobierno.

Heffernan, R. (2003). Prime Ministerial Predominance? Core Executive Politics in the UK. British Journal of Politics and International Relations, 5 (3), 347-372. Disponible en: https://doi.org/10.1111/1467-856X.00110.

Heywood, P. y Molina, I. (1997). La présidentialisation du système espagnol: La Moncloa. Revue française d'administration publique, 83, 447-458.

- (2000). A Quasi-Presidential Premiership: Administering the Executive Summit in Spain. En B. G. Peters, R. A. W. Rhodes y V. Wright (comps.). Administering the Summit. Administration of the Core Executive in Developed Countries (pp. 110-133). Nueva York: St. Martin's Press.

Inácio, M. y Llanos, M. (2015). The Institutional Presidency from a Comparative Perspective: Argentina and Brazil since the 1980s. Brazilian Political Science Review, 9 (1), 39-64. Disponible en: https://doi.org/10.1590/1981-38212014000200002.

Kölling, M. (2017). El centro de gobierno en Alemania entre Kanzlerdemokratie y Konsesusdemokratie. Ponencia presentada al XIII Congreso de la AECPA. Santiago de Compostela.

Lanzaro, J. (2016). El centro presidencial en Uruguay: 2005-2015. Revista Uruguaya de Ciencia Politica, 25 (2), 121-142.

Marsh, D., Richards, D. y Smith, M. (2003). Unequal Plurality: Towards an Asymmetric Power Model of British Politics. Government and Opposition, 38 (3), 306-332. Disponible en: https://doi.org/10.1111/1477-7053.t01-1-00017.

Méndez, J. L. (2014). La oficina presidencial en México, 2000-2014. ¿Certidumbre cooperativa o incertidumbre competitiva? Ponencia presentada al V Congreso Uruguayo de Ciencia Política. Montevideo.

Molina, I. (1998). La cima quasi-presidencial de un sistema parlamentario de gobierno: estructura y funcionamiento de Moncloa, manuscrito. Harvard: Harvard University, Department of Government.

Müller-Rommel, F. (1993). Ministers and the Role of the Prime Ministerial Staff. En J. Blondel y F. Müller-Rommel (comps.). Governing Together: The Extent and Limits of Joint Decision-Making in Western European Cabinets (pp. 131-152). Basingstoke: Macmillan. Disponible en: https://doi.org/10.1007/978-1-349-22936-9_6.

(1994). Management of Politics in the German Chancellor's Office. En G. Peters, R. A. W. Rhodes y V. Wright (comps.). Administering the Summit: Administration of the Core Executive in Developed Countries (pp. 81-100). Basingstoke: Macmillan.

- (2008). Prime Ministerial Staff in Post-Communist Central and Eastern Europe: A Role Assessment by Cabinet Ministers. Journal of Communist Studies and Transition Politics, 24 (2), 256-271. Disponible en: https://doi.org/10.1080/13523270802003095.

Olías, B. (1994). Los Gabinetes de los Presidentes del Gobierno en España. Politica y Sociedad, 16, 257-272.

Ortega, L. (1991). El Gabinete del Presidente del Gobierno. Documentación Administrativa, 226, 199-243. 
Paniagua, J. L. (2017). La Moncloa: una estructura presidencial para el Gobierno de España. Ponencia presentada al XIII Congreso de la AECPA. Santiago de Compostela.

Pfiffner, J. P. (1993). The President's Chief of Staff: Lessons Learned. Presidential Studies Quarterly, 23 (1), 77-102.

- (2011). Decision Making in the Obama White House. Presidential Studies Quarterly, 41 (2), 244-262. Disponible en: https://doi.org/10.1111/j.1741-5705.2011.03853.x.

Poguntke, T. y Webb, P. (eds.). (2005). The Presidentialization of Politics. A Comparative Study of Modern Democracies. Oxford: Oxford University Press. Disponible en: https://doi.org /10.1093/0199252017.001.0001.

Powell, J. (2011). The New Machiavelli. How to Wield Power in the Modern World. London: Vintage Books.

Prats i Català, J. y Villoria, M. (2011). El apoyo al Alto Gobierno: la coordinación y coherencia gubernamental. En M. Arenilla et al. (comps.). Fortalecimiento del Alto Gobierno: Aproximaciones conceptuales (pp. 55-90). Caracas: CLAD.

Rhodes, R. A. W. y Dunleavy, P. (eds.). (1995). Prime Minister, Cabinet and Core Executive. London: Macmillan. Disponible en: https://doi.org/10.1007/978-1-349-24141-5.

- y Tiernan, A. (2014a). Lessons in Governing: A Profile of Ministers' Chiefs of Staff. Melbourne: Melbourne University Press.

— (2014b). The Gatekeepers. Lessons from Prime Ministers's Chiefs of Staff. Melbourne: Melbourne University Press.

Santolaya Machetti, P. (1991). La experiencia de los gabinetes políticos en Derecho comparado. Documentación Administrativa, 226, 89-112.

Sullivan, T. (comp.). (2004). The Nerve Center. Lessons in Governing from White House Chiefs of Staff. College Station, TX: Texas A\&M Press.

Tiernan, A. y Pfiffner, J. P. (2014). Chiefs of Staff to Presidents and Prime Ministers: A Comparative Perspective. Ponencia presentada a la Conferencia anual de la American Political Science Association (APSA). Washington D. C. Disponible en: https://doi.org/10.2139/ ssrn. 2475602.

Truswell, E. y Atkinson, D. (2011). Supporting Heads of Government. A Comparison across Six Countries. London: Institute for Government.

Van Biezen, I. y Hopkin, J. (2005). The Presidentialization of Spanish Democracy: Sources of Prime Ministerial Power in Post-Franco Spain. En T. Poguntke y P. Webb (comps.). The Presidentialization of Politics: A Comparative Study of Modem Democracies (pp. 107-127). Oxford: Oxford University Press. Disponible en: https://doi.org/10.1093/0199252017 .003 .0005 .

Virgala Foruria, E. (1994). La organización interna del poder ejecutivo en los Estados Unidos: el presidente, el gabinete y la presidencia institucionalizada. Revista de Estudios Políticos, 83, 137-188.

Walcott, C. E. y Hult, K. M. (2005). White House Structure and Decision Making: Elaborating the Standard Model. Presidential Studies Quarterly, 35 (2), 303-318. Disponible en: https://doi.org/10.1111/j.1741-5705.2005.00250.x.

Whipple, C. (2017). The Gatekeepers. How the White House Chiefs of Staff Define Every Presidency. Nueva York: Crown. 


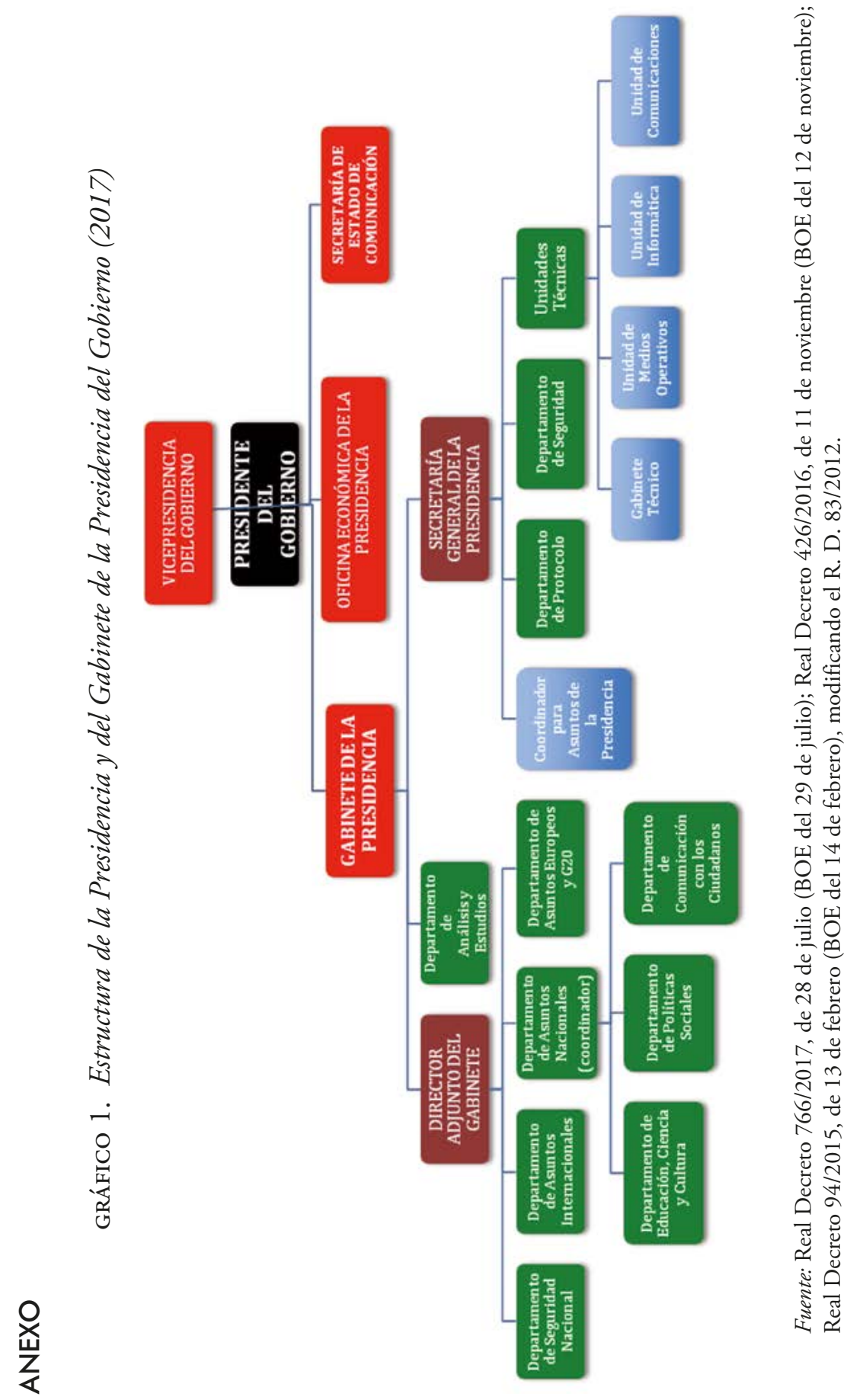




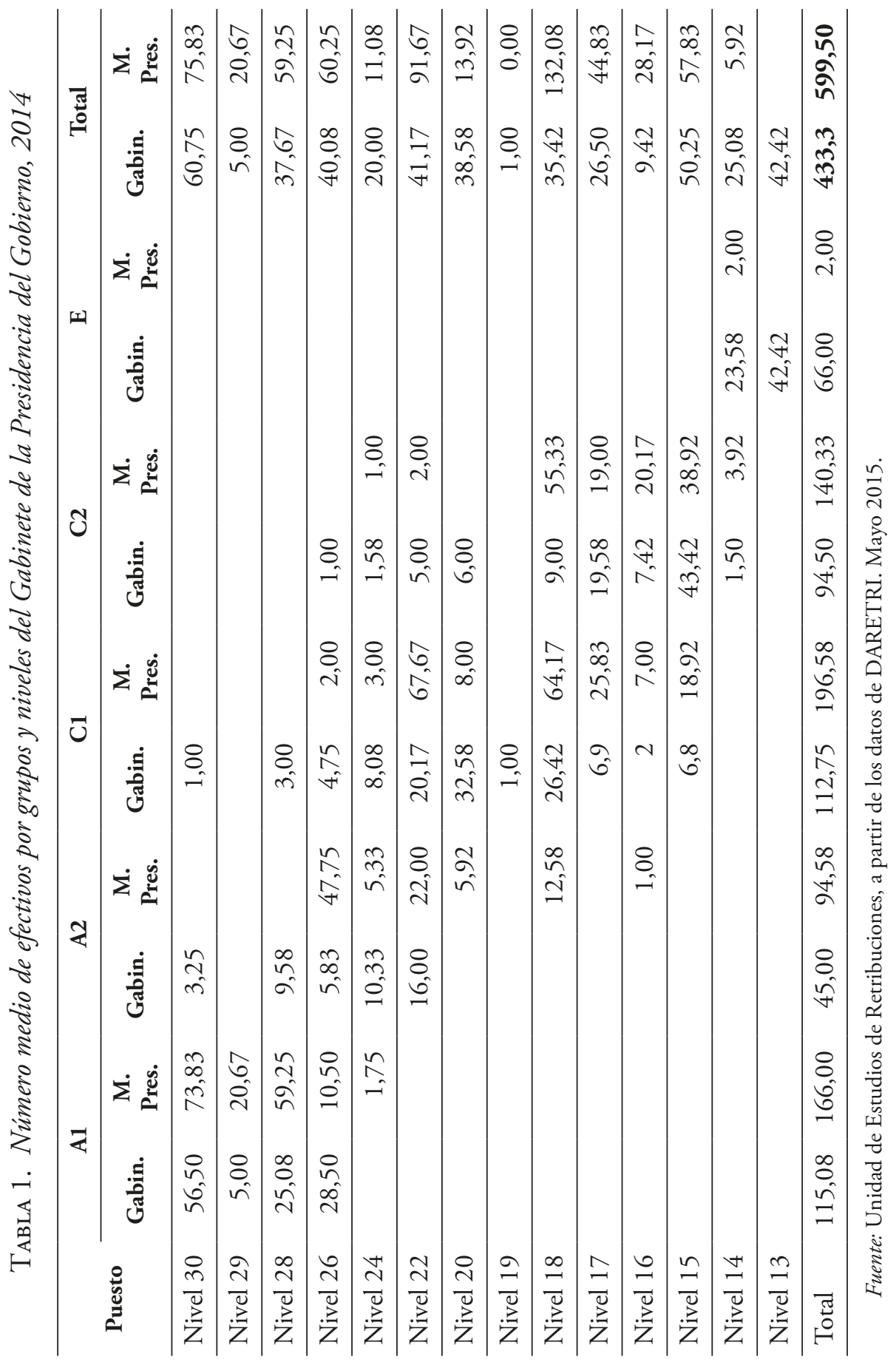




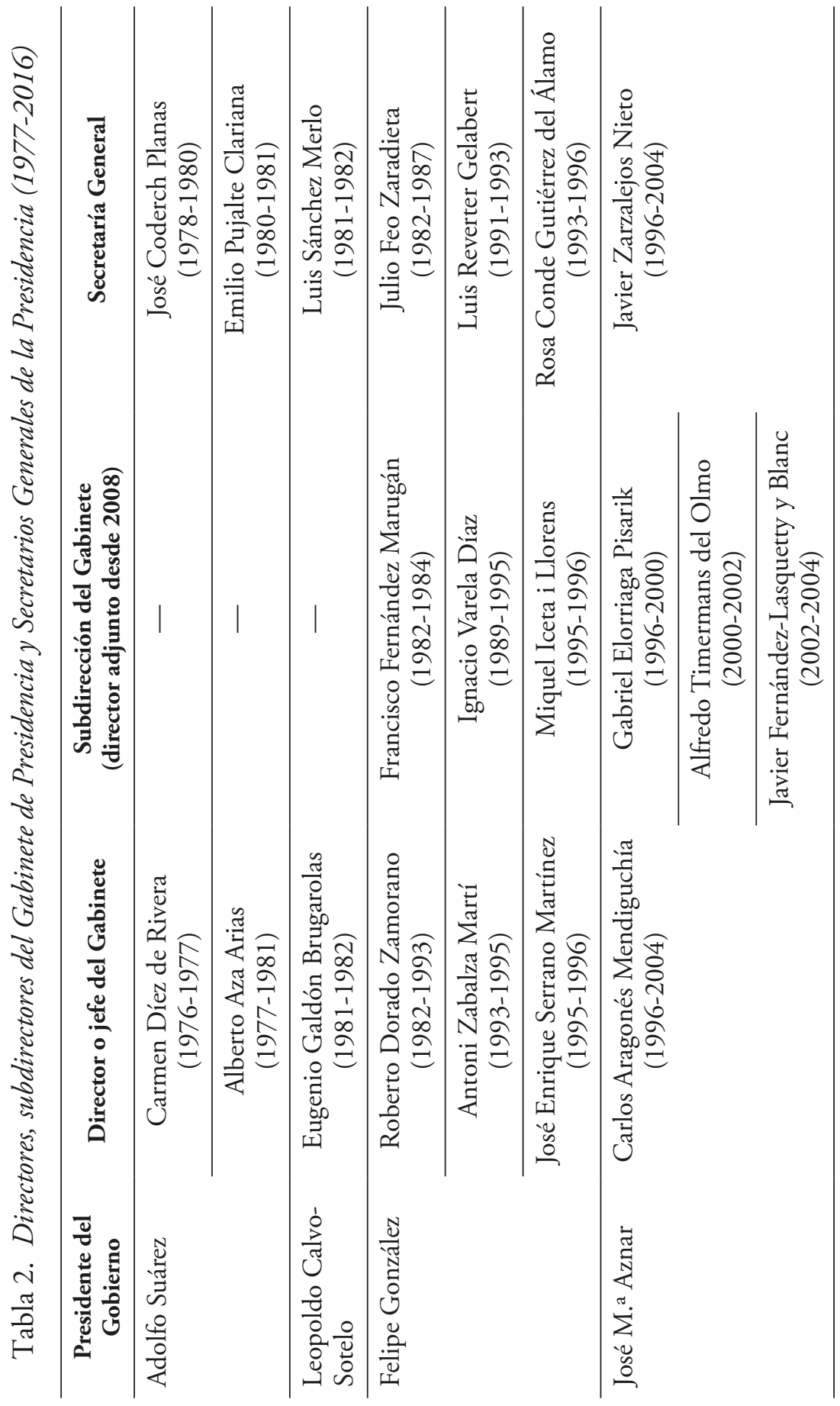




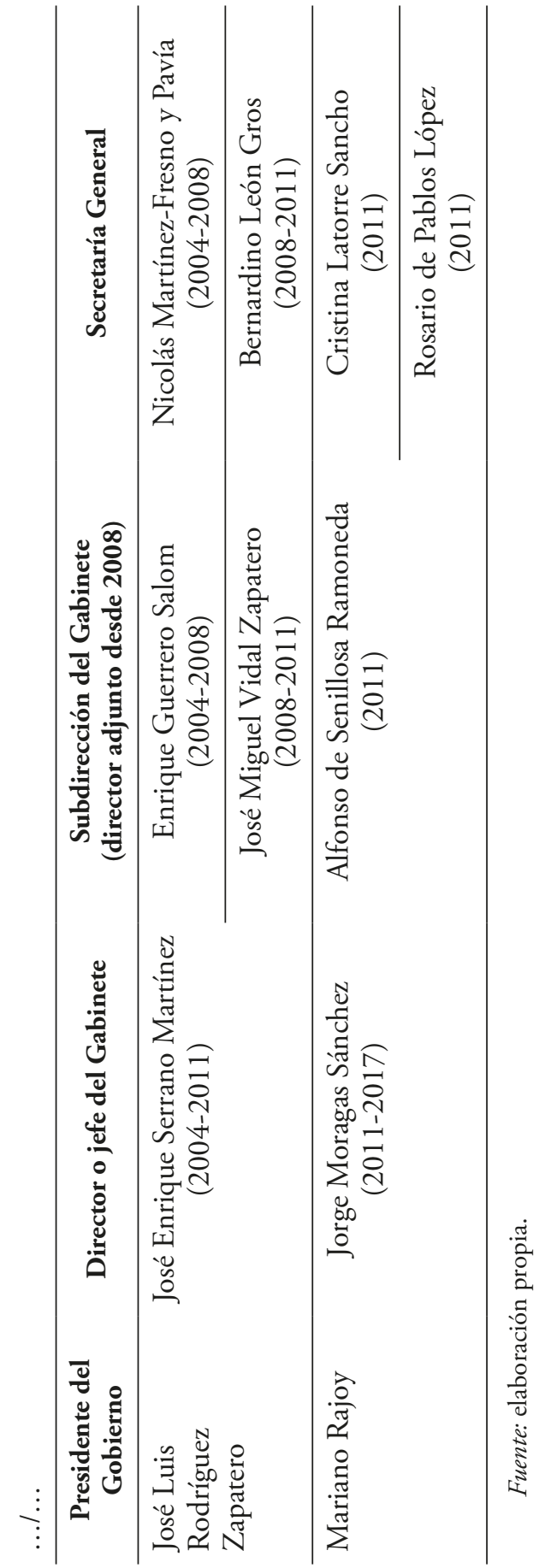


Tabla 3. Acceso total o parcial al presidente, según la discrecionalidad del jefe de Gabinete (1982)

\begin{tabular}{ll}
\hline \multicolumn{1}{c}{ Libre acceso } & \multicolumn{1}{c}{ Acceso parcial } \\
\hline Jefe del Estado & Ministros \\
Vicepresidente & Secretarios de Estado \\
Director Gabinete & Directores departamentos Gabinete \\
Jefe Secretaría Particular & Jefe Oficina Prensa Gabinete \\
Subjefe Secretaría Particular & Secretarios Ejecutiva Federal PSOE \\
Responsable Secretaría Personal & Secretarios generales regionales \\
Jefe Seguridad Personal & Alcaldes capitales y presidentes \\
Familiares y amigos (señalados de & Diputación \\
antemano) & Consejeros personales \\
Presidente PSOE y eventualmente tres & Portavoz grupo parlamentario \\
hombres & Secretario general partidos y sindicatos \\
Secretario general UGT & Presidentes comunidades autónomas \\
Presidente Cortes & Presidentes Parlamentos autónomos \\
Presidente Senado & Secretarios generales \\
Presidentes o jefes de Gobierno & Partidos socialistas de la Internacional \\
extranjeros & Socialista \\
Presidente internacional & Presidente CEOE \\
& Presidente de la A. Banca \\
& Presidente de la Conferencia Episcopal \\
\hline
\end{tabular}

Fuente: Gabinete de la Presidencia del Gobierno (s.f. [1982]: 27). 